\title{
Research
}

\section{Effects of educational interventions for self management of asthma in children and adolescents: systematic review and meta-analysis}

James P Guevara, Fredric M Wolf, Cyril M Grum, Noreen M Clark

\begin{abstract}
Objective To determine the effectiveness of educational programmes for the self management of asthma in children and adolescents.

Data sources Databases of the Cochrane Airways Group, PsychINFO, reference lists of review papers, and eligible studies.

Review methods Eligible studies were published randomised controlled trials or controlled clinical trials of educational programmes for the self management of asthma in children and adolescents that reported lung function, morbidity, self perception of asthma control, or utilisation of healthcare services. Eligible studies were abstracted, assessed for methodological quality, and pooled with fixed effects and random effects models.

Results 32 of 45 identified trials were eligible, totalling 3706 patients aged 2 to 18 years. Education in asthma was associated with improved lung function (standardised mean difference $0.50,95 \%$ confidence interval 0.25 to 0.75$)$ and self efficacy $(0.36,0.15$ to $0.57)$ and reduced absenteeism from school $(-0.14$, -0.23 to -0.04$)$, number of days of restricted activity $(-0.29,-0.33$ to -0.09$)$, and number of visits to an emergency department $(-0.21,-0.33$ to -0.09$)$. When pooled by the fixed effects model but not by the random effects model, education was also associated with a reduced number of nights disturbed by asthma. The effect on morbidity was greatest among programmes with strategies based on peak flow, interventions targeted at the individual, and participants with severe asthma.

Conclusions Educational programmes for the self management of asthma in children and adolescents improve lung function and feelings of self control, reduce absenteeism from school, number of days with restricted activity, number of visits to an emergency department, and possibly number of disturbed nights. Educational programmes should be considered a part of the routine care of young people with asthma.
\end{abstract}

\section{Introduction}

Educational programmes for the self management of asthma in children have been developed to improve healthcare practices, reduce morbidity, and lower the costs of care. ${ }^{12}$ Experts have recommended that programmes be based on sound theoretical understandings of change in behaviour and that they employ strategies designed to improve knowledge, skills, and feelings of self control. ${ }^{3}$ Not all programmes have been conceptually based, however, and many are an ad hoc set of messages and skills incorporated into didactic lectures by clinicians. ${ }^{4}$ Several conceptually based programmes have been the subject of rigorous evaluation, and results show that educating patients about their asthma can improve self management practices. ${ }^{2}$

Although it is clear that education by only the transfer of information is ineffective, the effectiveness of educating children with asthma in self management is unclear. ${ }^{5}$ Programmes aimed at adults with asthma that include self monitoring, regular medical review, and an asthma action plan do seem to reduce morbidity and the use of healthcare resources, but a meta-analysis of self management in children found no such association. ${ }^{67}$ The meta-analysis was limited to trials published before 1992, and several rigorous evaluations have been subsequently completed. We aimed to estimate the effectiveness of educational programmes in self management on clinical outcomes in children and adolescents with asthma by incorporating more recent studies.

\section{Participants and methods}

We searched the Cochrane Airways Group's special register of controlled trials, composed of references from Medline (1966-98), Embase (1980-98), and CINAHL (1982-98), and hand searched airways related journals, using the search terms asthma OR wheez* AND education* OR self management OR self-management AND placebo* OR trial* OR random* OR double-blind OR double blind OR single-blind OR single blind OR controlled study OR comparative study. PsychINFO (to 1998) was also searched to identify trials published in the educational or behavioural science literature by using the Cochrane Schizophrenia Group's search strategy: asthma* OR ASTHMA- in DE OR wheez* OR [(BRONCHIAL*) near (HYPER-REACTIV* or HYPERREACTIV*)]. The reference lists from relevant review articles and all eligible studies were also hand searched. ${ }^{378}$

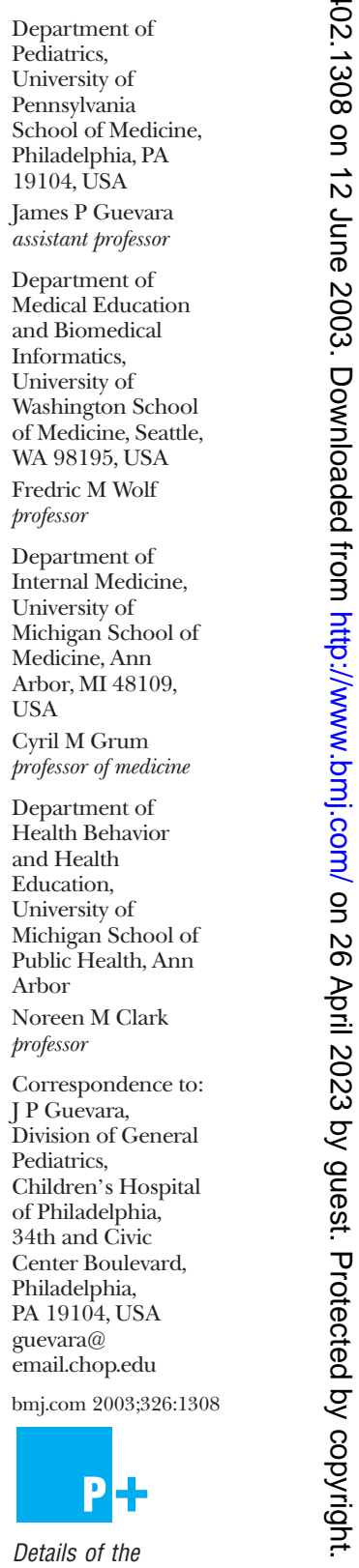
on bmj.com 


\section{Selection and data abstraction}

Studies published in any language were eligible if they fulfilled the following criteria: were randomised controlled trials or controlled clinical trials; included children aged 2 to 18 years with asthma; incorporated educational interventions in self management related to prevention of asthma, management of asthma attacks, or development of social skills; reported outcomes of interest. Studies were excluded if they included participants with pulmonary diagnoses other than asthma, enrolled children aged less than 2 years, lacked a concurrent control population, used nonstandard educational interventions, or lacked outcomes of interest.

We screened the title and abstract of citations obtained through the search strategy and obtained the full text of potentially eligible studies. Non-English language articles were translated into English. Two investigators independently assessed each article for eligibility, and disagreement was settled by consensus. All eligible studies were abstracted on forms. To clarify procedures or to obtain missing data, corresponding authors were contacted by post or email.

Validity assessment and study characteristics Study quality was based on whether assignment of intervention was concealed before enrolment. ${ }^{9}$ Trials were categorised as adequate, unclear, or clearly inadequate. In addition, we judged whether systematic differences in care, withdrawals, or outcome assessment were evident between treatment and control groups. ${ }^{910}$

For each eligible study we abstracted information on randomisation, allocation concealment, participants, settings, follow up procedures, characteristics of educational interventions (type, strategy, number of sessions, duration, and educational tools), and outcomes. Outcomes of interest were forced expiratory volume in one second $\left(\mathrm{FEV}_{1}\right)$ and peak expiratory flow rate as measures of lung function, number of days absent from school, number of days of restricted activity, number of disturbed nights, self efficacy scales (including coping scores or health locus of control scales), symptom scores, number of visits to an emergency department, and hospitalisations. The severity of asthma was assessed from trial self report, examination of mean baseline $\mathrm{FEV}_{1}$ or peak expiratory flow rate, or chronicity of asthma symptoms at baseline. ${ }^{11} 12$ Studies were categorised as moderatesevere if participants had severe asthma, mildmoderate if participants had mild or moderate asthma, or unclear if severity was not reported and could not be deduced.

\section{Quantitative data synthesis}

Because measures were reported with different scales or time intervals, we used the standardised weighted mean difference with 95\% confidence interval to estimate a pooled effect size for each outcome of interest. (The standardised weighted mean difference, reported in units of standard deviation, converts the difference in mean values for each trial into a common metric.) Data were pooled with both fixed effects and random effects models. ${ }^{13}{ }^{14}$ For consistency we report in both the text and the figures the standardised weighted mean difference based on the fixed effects model. When effect sizes differ statistically, we report in the text only the pooled effect sizes from the random effects model. Measures of lung function were also back translated by multiplying the standardised weighted mean difference from the fixed effects model by the standard deviation of the control group for $\mathrm{FEV}_{1}$ and peak expiratory flow rate. Homogeneity of effect sizes was assessed by the $Q$ statistic. ${ }^{14}$ Funnel plot asymmetry was examined for publication bias. ${ }^{15}$

If continuous outcomes were reported without measures of variance, we imputed pooled standard deviations using the $t$ statistic formula. ${ }^{16}$ If the $t$ statistic was not reported, we chose the $t$ statistic corresponding to the exact $\mathrm{P}$ value with the appropriate degrees of freedom. If the $t$ statistic and exact $\mathrm{P}$ value were not reported, we chose the $t$ statistic corresponding to $\mathrm{P}=0.05$ (for a reported $\mathrm{P}<0.05$ ) or $\mathrm{P}=0.50$ (for a reported $\mathrm{P}>0.05)$ with the appropriate degrees of freedom. ${ }^{17}$ Studies with missing point estimates were excluded from the pooled estimates, but the results were reported qualitatively.

\begin{tabular}{|c|c|c|c|c|c|c|c|c|}
\hline Study & $\begin{array}{l}\text { No in education } \\
\text { group }\end{array}$ & $\begin{array}{l}\text { Mean } \\
\text { (SD) }\end{array}$ & $\begin{array}{l}\text { No in control } \\
\text { group }\end{array}$ & $\begin{array}{l}\text { Mean } \\
\text { (SD) }\end{array}$ & \multicolumn{2}{|c|}{$\begin{array}{l}\text { Standardised weighted } \\
\text { mean difference } \\
(95 \% \mathrm{Cl} \text { fixed })\end{array}$} & $\begin{array}{c}\text { Weight } \\
(\%)\end{array}$ & $\begin{array}{l}\text { Standardised weighted } \\
\text { mean difference } \\
\text { (95\% Cl fixed) }\end{array}$ \\
\hline Carswell 1989w2 & 43 & $109.00(19.00)$ & 43 & $100.00(19.00)$ & & - & 34.3 & $0.47(0.04$ to 0.90$)$ \\
\hline Toelle $1993^{\text {w29 }}$ & 60 & $2.13(0.51)$ & 50 & $1.90(0.47)$ & & $\rightarrow-$ & 43.6 & 0.46 (0.08 to 0.84$)$ \\
\hline Subtotal $(95 \% \mathrm{Cl})$ & 103 & & 93 & & & $<$ & 77.9 & $0.47(0.18$ to 0.75$)$ \\
\hline \multicolumn{9}{|c|}{ Moderate-severe asthma } \\
\hline Christiansen $1997^{\mathrm{W}}$ & 27 & $331.37(53.52)$ & 15 & $313.53(40.63)$ & & $=$ & 15.6 & $0.35(-0.28$ to 0.99$)$ \\
\hline Subtotal $(95 \% \mathrm{Cl})$ & 27 & & 15 & & & & 15.6 & $0.35(-0.28$ to 0.99$)$ \\
\hline \multicolumn{9}{|l|}{ Unclear severity } \\
\hline Weingarten $1985^{\mathrm{w} 30}$ & 11 & $276.60(33.86)$ & 9 & $232.80(33.86)$ & & $\longrightarrow$ & 6.6 & 1.24 (0.26 to 2.22$)$ \\
\hline Subtotal $(95 \% \mathrm{Cl})$ & 11 & & 9 & & & - & 6.6 & 1.24 (0.26 to 2.22$)$ \\
\hline Total $(95 \% \mathrm{Cl})$ & 141 & & 117 & & & $\diamond$ & 100 & $0.50(0.25$ to 0.75$)$ \\
\hline \multicolumn{5}{|c|}{ Test for heterogeneity: $\chi^{2}=2.44, d f=3, P=0.49$} & -2 & 0 & 4 & \\
\hline \multicolumn{5}{|c|}{ Test for overall effect: $z=3.90, P=0.0001$} & ontro & Favours & & \\
\hline
\end{tabular}

Fig 1 Effect of educational programmes in self management of asthma on lung function. Lung function was reported as changes in absolute forced expiratory volume in one second or peak expiratory flow rate or as changes in percentage predicted peak flow expiratory flow rate 


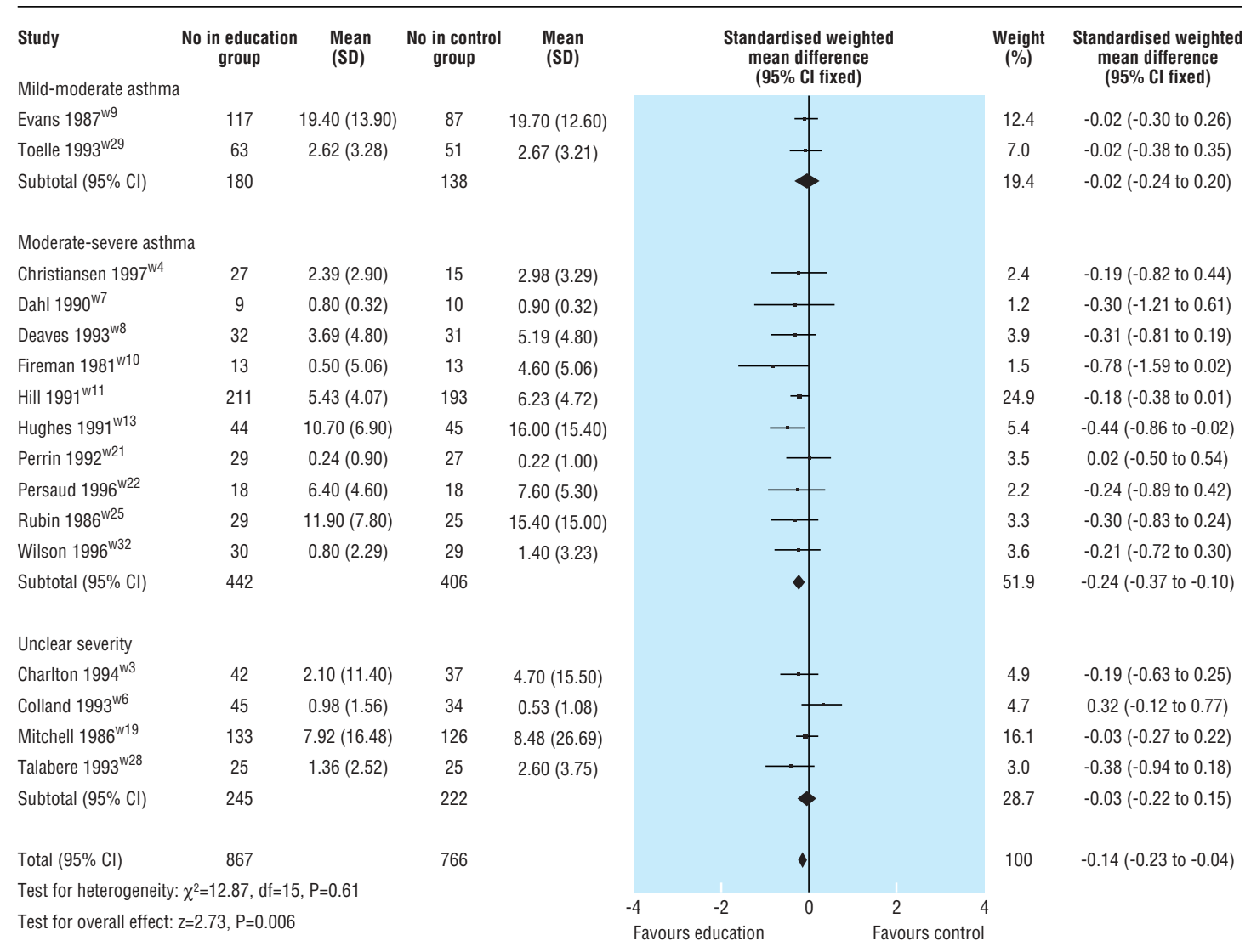

Fig 2 Effect of educational programmes in self management of asthma on absenteeism from school. Absenteeism refers to number of days absent from school because of asthma or other causes, reported as mean number of days a month, two months, six months, 10 months, or year

Subgroups were analysed to estimate the magnitude of the effect of study quality and programme components on outcome measures. For study quality we limited studies to randomised controlled trials, those with adequate concealment of allocation, and those without systematic differences in withdrawals. For programme components we stratified on type of intervention (individual $v$ group), intensity of intervention (single session $v$ multiple sessions), and self management strategy (peak flow based $v$ symptom based).

\section{Results}

Overall, 45 of 318 identified studies were potentially eligible. Thirteen were excluded owing to absence of a control population $(n=2)$, inclusion of children under 2 years old $(n=2)$, use of non-standard or information only educational interventions $(n=4)$, inclusion of children with conditions other than asthma $(n=1)$, and absence of outcomes of interest $(n=4)$. This left 32 trials totalling 3706 children and adolescents with asthma (see table A on bmj.com). Most were relatively small randomised controlled trials and enrolled children with severe asthma. Fifteen trials enrolled adolescents aged 13 to 18 years, and 12 enrolled children aged 2 to 5 years; no study stratified data on age. The educational programmes were diverse and targeted children, parents, or both. Most had programmes with multiple sessions and symptom based strategies. Methodologi- cal quality on the basis of allocation concealment was adequate in only $12(38 \%)$ studies, but many studies contained insufficient information to determine study quality (see table B on bmj.com). Few studies had systematic differences in care or withdrawal.

\section{Quantitative data synthesis}

Four trials (258 patients) had complete data on measures of lung function (fig 1). Education was associated with moderate improvement on a combined measure of lung function $(0.50,0.25$ to 0.75$)$ and on individual measures of $\mathrm{FEV}_{1}(0.46,0.08$ to 0.84$)$ and peak expiratory flow rate $(0.53,0.19$ to 0.86$)$. This translated into a 0.24 litre increase in $\mathrm{FEV}_{1}$ and a 9.5\% increase in percentage predicted peak expiratory flow rate associated with education. Three additional trials (192 patients) could not be pooled owing to missing point estimates; these studies reported no effect of education on lung function. No evidence of publication bias was found (intercept $-0.9,90 \%$ confidence interval -2.7 to 4.5 ).

Eighteen trials (1649 patients) had complete data on measures of morbidity. Education was associated with a modest reduction in absenteeism from school $(-0.14,-0.23$ to -0.04 ; fig 2$)$. One trial (84 patients) could not be pooled owing to missing point estimates, but it did report less absenteeism from school in the group that received the educational intervention. Education was also associated with a reduction in number of days of restricted activity $(-0.29,-0.49$ to -0.08$)$ and number of disturbed nights $(-0.34,-0.62$ to -0.05$)$. 


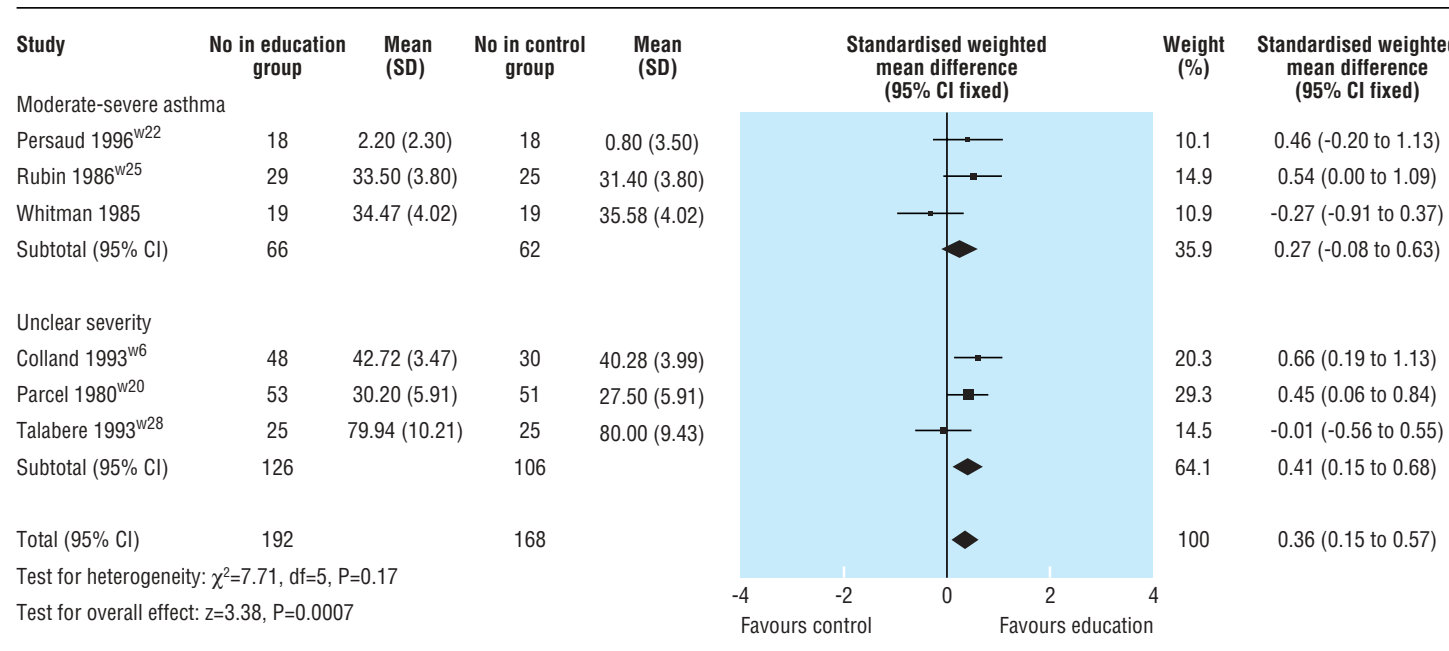

Fig 3 Effect of educational programmes in self management of asthma on self efficacy measures. Self efficacy measures were reported as coping scores or health locus of control scales

Heterogeneity was found among trials pooled for number of nights disturbed by asthma $\left(\chi^{2}=11.2, \mathrm{df}=2\right.$, $\mathrm{P}=0.004$ ) but not for other morbidity outcomes. The pooled estimate from the random effects model for number of disturbed nights was not significant $(-0.39$, -1.07 to 0.28$)$. Outcomes were generally stronger among trials of moderate-severe asthma than among those of mild-moderate asthma. No evidence of publication bias was found for any measure of morbidity.

Nine trials (522 patients) reported complete data on measures of self perception of asthma control. We found a moderate improvement in self efficacy $(0.36$, 0.15 to 0.57 ; fig 3 ). Four additional trials (356 patients) could not be pooled owing to missing point estimates; education was shown as beneficial in three. Education had no effect on symptom scores. No statistical heterogeneity was found for self efficacy, but it was found for symptom scores $\left(\chi^{2}=6.7, \mathrm{df}=3, \mathrm{P}=0.08\right)$. Results were, however, consistent across both models. No evidence of publication bias was found.

Eighteen trials (1899 patients) reported complete data on measures of utilisation of healthcare services. Education was associated with a modest reduction in number of visits to an emergency department $(-0.21$, -0.33 to -0.09 ; fig 4 ). Two trials (127 patients) could not be pooled owing to missing point estimates; one reported a reduction in number of visits. Education had no effect on hospitalisations. Utilisation outcomes

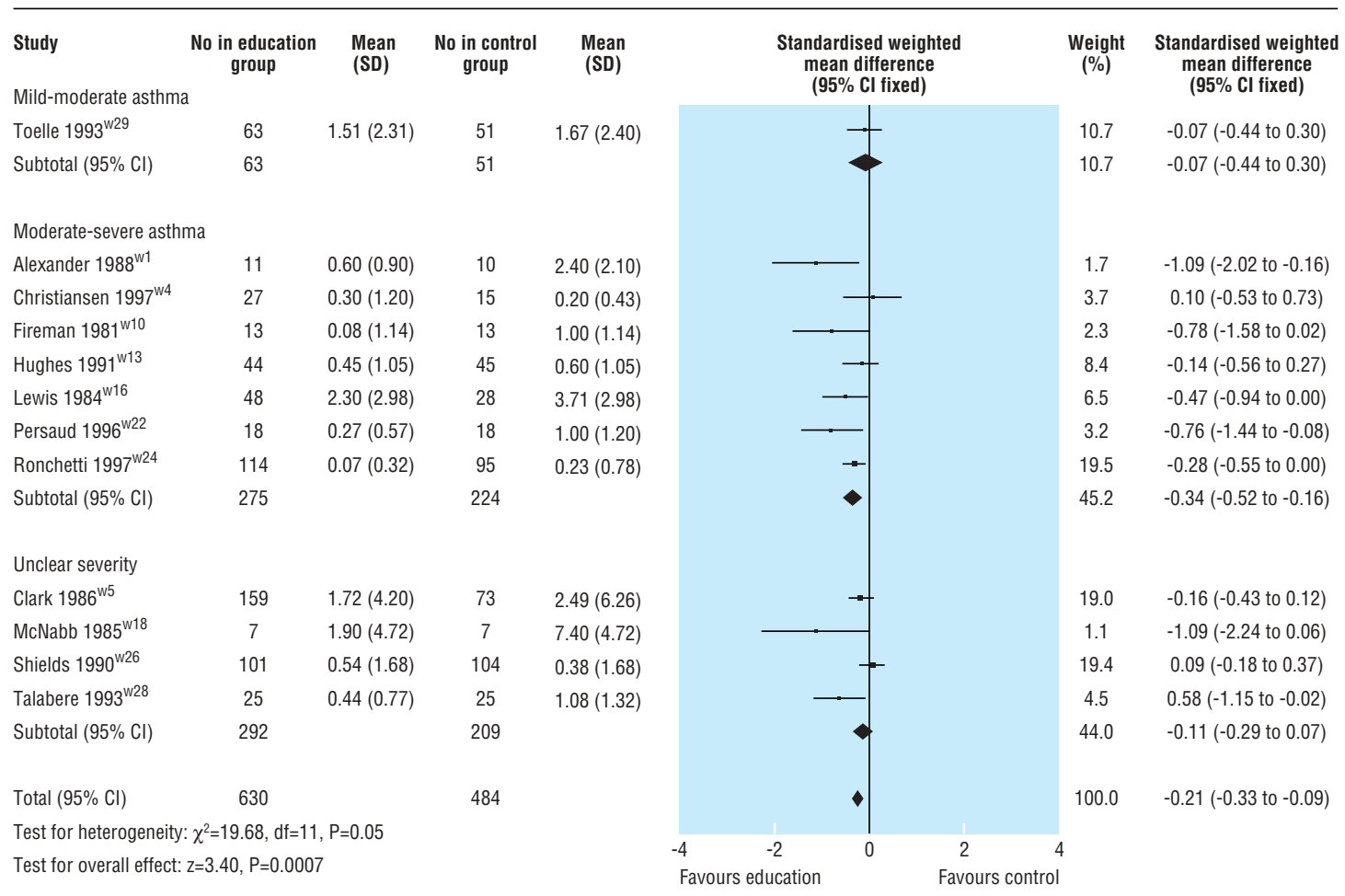

Fig 4 Effect of educational programmes in self management of asthma on number of visits to an emergency department. Visits refers to hospital emergency departments, reported as mean number of visits every three months, four months, or year 
were stronger among trials of moderate-severe asthma than among those of mild-moderate asthma. Possible publication bias was found for hospitalisations (intercept $-3.0,-4.7$ to -1.3 ) but not for visits to an emergency department.

\section{Subgroup analyses}

To assess the impact of study quality, analyses were restricted to studies of higher quality. We found similar estimates for lung function, self efficacy, morbidity, and utilisation of healthcare services when studies were limited to those with adequate allocation concealment, randomised controlled trials, or those without systematic differences in withdrawals. To assess programme characteristics, we stratified analyses on self management strategy and found that programmes based on peak flow had the greatest improvement in lung function and the greatest reductions in morbidity measures. We also stratified on intervention type and found that programmes targeted at the individual had the greatest reductions in morbidity measures, whereas programmes targeted at a group had the greatest reduction in hospitalisations. Finally, we stratified on intervention intensity and found that programmes comprising single sessions had the greatest reductions in morbidity measures, whereas those comprising multiple sessions had the greatest improvement in self efficacy and the greatest reduction in number of visits to an emergency department. No studies involved direct comparisons between different educational components.

\section{Discussion}

Educational programmes for the self management of asthma in children and adolescents were associated with modest to moderate improvement in many outcome measures, including lung function, self efficacy, absenteeism from school, number of days of restricted activity, number of visits to an emergency department, and possibly nights disturbed by asthma. Education seemed to be as effective among studies of mild-moderate asthma as among those of moderatesevere asthma; for many morbidity outcomes, however, effects of education were strongest in studies enrolling patients with more severe asthma. Programmes with strategies based on peak flow showed the strongest effects on morbidity measures, as did programmes with interventions aimed at the individual. These results should be interpreted cautiously given the lack of direct comparisons in primary studies. The results obtained among studies considered to be of higher quality generally supported the main findings.

Although our results support the findings observed in adults with asthma, our results differed from a previously published meta-analysis of asthma education in children. ${ }^{6}$ This review, involving 11 trials published between 1981 and 1991, found no benefit of education, as the authors were only able to pool between three and five studies for any one outcome. ${ }^{7}$ This may have limited their statistical power to identify small effects. Our review included these 11 trials and an additional 21 trials, nine of which were published between 1980 and 1991 and either were not identified or were excluded by these authors. We were also able to evaluate a wider range of outcomes and to provide

\section{What is already known on this topic}

Evidence on the effectiveness of educating children about their asthma has been conflicting

A meta-analysis found no evidence of reduction in morbidity or utilisation of healthcare resources associated with educational programmes

\section{What this study adds}

Educational programmes in the self management of asthma improve lung function and self efficacy and reduce morbidity and utilisation of healthcare resources

Such programmes should be part of the routine care of young people with asthma

tentative estimates of important comparisons between subgroups.

Our study has several limitations. Firstly, the effect of education on most morbidity measures was limited by the paucity of studies reporting these outcomes. For instance, quality of life-considered an important outcome in asthma-was not reported by any eligible trial. Secondly, many studies did not adequately report methods and results. This limited our ability to estimate the effects of study quality or to pool data. Thirdly, we may not have identified all relevant trials of asthma education; formal tests of funnel plot asymmetry showed publication bias only for hospitalisations, a non-significant outcome. Fourthly, there were insufficient studies and a lack of direct comparisons to reliably estimate subgroup effects. Our subgroup analyses should therefore be interpreted with caution.

Our study has important implications for practice and research. Incorporating educational programmes for self management into the routine care of children with asthma may significantly improve outcomes. Although selection of a programme may depend on cost and availability, efforts should be made to incorporate models that are known to work. Priority should be given to patients with severe asthma, and education should be provided long term to account for changing needs. Future studies should test alternative components directly to determine their relative effectiveness-for example, studies should focus on morbidity measurements and quality of life and directly compare strategies based on peak flow with those based on symptoms and compare strategies aimed at the individual with those aimed at the group. Assessment of the impact of asthma education would be enhanced if trials reported in a uniform way. ${ }^{18}$ Studies should be conducted over longer periods, report age stratified outcomes, and be adequately powered to determine clinically relevant effects.

A detailed version of this systematic review is published in the Cochrane database of systematic reviews (www.updatesoftware.com/abstracts/ab000326.htm). We thank the Cochrane Airways Review Group, including Steve Milan, Francine Ducharme, Anna Bara, Jane Dennis, Christopher Cates, Mike McKean, Karen Blackhall, and Paul Jones for assistance in identifying and translating trials, helpful comments, and editorial assistance.

Contributors: JPG participated in the identification and selection of studies, data extraction, data analysis and interpret- 
ation, writing and revising the manuscript, and correspondence with authors. FMW initiated and designed the protocol, participated in the identification and selection of studies, data analysis and interpretation, and writing and revising the paper; he will act as guarantor for the paper. CMG participated in the identification and selection of studies and writing and revising the manuscript. NMC participated in the design of the protocol and writing and revising the manuscript.

Funding: National Institutes of Health Fogarty International Center (grant No NIH 1 F06 TW02123) and National Heart, Lung, and Blood Institute (grant No NIH 1 K07 HL 03046).

Competing interests: None declared.

1 Hurd SS, Lenfant C. The National Heart, Lung, and Blood Institute Asthma Program. Chest 1992;101:359-61S.

2 Clark NM. Asthma self-management education: research and implications for clinical practice. Chest 1989;95:1110-3.

3 Clark NM, Starr-Schneidkraut NJ. Management of asthma by patients and families. Am J Respir Crit Care Med 1994;149:54S-66S

4 Clark NM, Gong M. Management of chronic disease by practitioners and patients: are we teaching the wrong things? BMJ 2000;320:572-5.

5 Gibson PG, Coughlan J, Wilson AJ, Hensley MJ, Abramson M, Bauman A et al. Limited (information only) patient education programs for adults with asthma. Cochrane Library. Issue 1. Oxford: Update Software, 2000.

6 Gibson PG, Coughlan J, Abramson M, Bauman A, Hensley MJ, Walters $\mathrm{EH}$, et al. The effects of self-management education and regular practitioner review in adults with asthma. Cochrane Library. Issue 2. Oxford: Update Software, 1998.
7 Bernard-Bonnin A, Stachenko S, Bonin D, Charette C, Rousseau E. Selfmanagement teaching programs and morbidity of pediatric asthma: a meta-analysis J Allergy Clin Immunol 1995:95:34-41.

8 Clark N, Gotsch A, Rosenstock I. Patient, professional, and public education on behavioral aspects of asthma: a review of strategies for change and needed research. J Asthma 1993;30:241-55

9 Clarke M, Oxman A. The cochrane reviewers' handbook 4.1.5. Cochran Library. Issue 2. Oxford: Update Software, 2002.

10 Greenhalgh T. How to read a paper: assessing the methodological quality of published papers. BMJ 1997:315:305-8.

11 American Thoracic Society. Lung function testing: selection of reference values and interpretative strategies. Am Rev Respir Dis 1991;144:1202-18.

2 National Asthma Eduction Progiam, Reu and and management of asthra. Bethesda, MD: National Instimutes of Healt

13 Hasselblad V, Hedges L. Meta-analysis of screening and diagnostic tests. Psychol Bull 1995;117:167-78.

14 DerSimonian R, Laird N. Meta-analysis in clinical trials. Control Clin Trials $1986 ; 7: 177-88$

15 Egger M, Smith GD, Schneider M, Minder C. Bias in meta-analysis detected by a simple, graphical test. BMJ 1997;315:629-34.

16 Rosenthal R. Meta-analytic procedures for social research. Newbury Park, CA: Sage, 1991:13-7.

17 Wolf FM, Guevara JP. Imputation of missing data in systematic reviews: so what is the standard deviation? [Abstract P-007.] Proceedings of the ninth International Cochrane Colloquium. Lyon, France. Ninth International Cochrane Colloquium: BMC Meeting Abstracts: 2001, 1:pa007. www.biomedcentral.com/abstracts/cochrane/1/pa007/

18 Begg C, Cho M, Eastwood S, Horton R, Moher D, Olkin I, et al. ImprovBegg C, Cho M, Eastwood S, Horton R, Moher D, Olkin I, et al. Improving the quality of reporting of randomized

(Accepted 14 March 2003) 